\title{
Asociación entre hipercolesterolemia y colesterolosis o litiasis vesicular
}

\author{
Association between hypercholesterolemia and cholesterolosis or vesicular lithiasis \\ Carlos David Vilela Desposorio ${ }^{1, a ; 2, b, c}$, Giancarlo Moisés Aurazo Zapata ${ }^{3, d}$, María Julissa Abanto Vaella ${ }^{4, d}$
}

\section{RESUMEN}

Objetivos: Determinar si existe asociación entre hipercolesterolemia y colesterolosis o litiasis vesicular. Material y métodos: Estudio de serie de casos comparativo, retrospectivo, realizado entre enero del 2014 y diciembre del 2015, en el Hospital Belén de Trujillo-Perú. Formaron parte del estudio todos aquellos casos productos de colecistectomía que tuvieron un perfil lipídico. Se revisaron 1069 historias clínicas en las que se encontraron 143 casos que tenían perfil lipídico. Resultados: El rango de edad varió entre 17 y 95 años con una edad promedio de 50 años. De estos, 36 casos $(25,2 \%)$ tenían colesterolosis. La relación varón:mujer fue 1:2,6 y una edad promedio de 44,5 años. Tuvieron colecistolitiasis 119 casos $(83,2 \%)$, con una relación varón:mujer 1:3,1 y una edad promedio de 48 años. Hubo hipercolesterolemia ( $\geq 200 \mathrm{mg} / \mathrm{dl})$ en 51 casos (35,7\%). En 29 (20,3\%) casos se observó colesterolosis con colecistolitiasis y $7(4,9 \%)$ pacientes tuvieron colesterolosis acalculosa. No se encontró asociación entre hipercolesterolemia y colesterolosis $(p=0,284)$, ni con litiasis vesicular $(p=0,169)$. Conclusiones: No existe asociación estadísticamente significativa entre hipercolesterolemia con colesterolosis, ni con litiasis vesicular.

PALABRAS CLAVE: Vesícula biliar, colecistolitiasis, hipercolesterolemia. (Fuente: DeCS BIREME).

\section{SUMMARY}

Objective: To determine if an association hypercholesterolemia and cholesterolosis or gallbladder lithiasis does exist. Methods: AA case series study was conducted between January 2014 and December 2015 in Hospital Belén, TrujilloPerú. Patients were those in whom a cholecystectomy had been performed (1069) and a lipid profile was available (143). Results: Age range was 17-95 years with a mean age of 50; $36(25.2 \%)$ had cholesterolosis, male to female ratio was 1:2,6. Gallbladder lithiasis was found in 119 cases (83.2\%), male to female ratio is 1:3.1 and a mean age of 48. Hypercholesterolemia ( $\geq 200 \mathrm{mg} / \mathrm{dl}$ ) was found in 51 cases $(35.7 \%) ; 2920.3 \%$ cholesterolosis with gallbladder lithiasis and 7 (4.9\%) had acalculous cholesterolosis. No association was found between hypercholesterolemia and cholesterolosis $(\mathrm{p}=0.284)$ nor with gallbladder lithiasis $(\mathrm{p}=0.169)$. Conclusions: No significant association between hypercholesterolemia and cholesterolosis or gallbladder lithiasis was found.

KEYWORDS: Gallbladder, cholecystolithiasis, hypercholesterolemia. (Source: MeSH NLM).

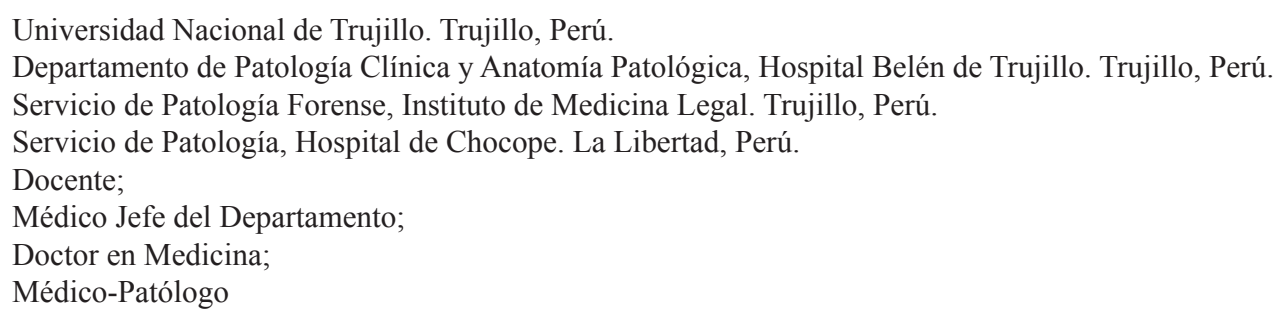


Se denomina colesterolosis de la vesícula biliar a la acumulación, en la lámina propia o corion de la mucosa, de histiocitos cargados de lípidos (esteres de colesterol y triglicéridos). La colesterolosis puede manifestarse de varias formas $(1,2)$ : Colesterolosis difusa, distribuida a lo largo de todo el epitelio vesicular, con patrones granular o reticular, color amarillo; pólipos de colesterol, o combinación de colesterolosis difusa y pólipos de colesterol, y colesterolosis focal (2).

En la colesterolosis, el colesterol se acumula dentro de los histiocitos de la mucosa de la vesícula por absorción de colesterol de la bilis, mediante endocitosis y por alteraciones en el transporte de colesterol desde la pared hacia el lumen vesicular (3$5)$.

La bilis saturada de colesterol induce la formación de cristales de colesterol y cálculos (3-7); sin embargo, se ha demostrado que pacientes obesos y no obesos pueden tener una bilis no saturada de colesterol y un tiempo de nucleación (inicio de formación de cristales) biliar normal (6), en consecuencia, el desarrollo concomitante de cálculos y colesterolosis vesicular no se asocia necesariamente a una bilis supersaturada. Del mismo modo, Méndez-Sánchez N. et al., (7), no pudieron demostrar asociación entre colesterolosis vesicular o la formación de cálculos biliares y niveles elevados de colesterol plasmático.

La incidencia de la colesterolosis es variable dependiendo de las series y de la indicación que haya tenido la colecistectomía, alcanzando hasta $30 \%$ en pacientes con litiasis y hasta $50 \%$ en pacientes obesos mórbidos (8-11). Algunos de los factores predictores de colesterolosis son: el índice de masa corporal, la circunferencia abdominal y la proteína $\mathrm{C}$ reactiva de alta sensibilidad (8). También existen estudios que han asociado la presencia de colesterolosis a hipercolesterolemia $(12,13)$.

Los cristales de colesterol en la bilis se forman cuando en el proceso cantidades de agua y lecitina no son suficientes para mantener la fluidez de la bilirrubina y el colesterol, por tanto, los cristales precipitan y estos más tarde darán lugar a los cálculos (14).

Beltran et al. (15), encontraron que hipercolesterolemia se asocia a colelitiasis, pero no a colesterolosis, encontrando que el $82,5 \%$ de los pacientes con colesterol normal tenían colesterolosis, mientras que, la mayoría de los pacientes $(90,6 \%) \sin$ colesterolosis tenía niveles elevados de colesterol.

Estos datos controversiales nos motivaron a llevar a cabo el presente estudio cuyo objetivo fue determinar si existe asociación entre hipercolesterolemia y colesterolosis o litiasis de la vesícula biliar.

\section{MATERIAL Y MÉTODOS}

Estudio de serie de casos comparativo, retrospectivo. El universo muestral estuvo constituido por todos los pacientes atendidos en el servicio de cirugía general cuyas piezas quirúrgicas post colecistectomía, fueron remitidas para su estudio al servicio de Patología Quirúrgica, del Hospital Belén de Trujillo, desde enero del 2014 hasta diciembre del 2015.

Criterios de inclusión:

- Piezas operatorias de pacientes operados en el hospital Belén.

- Piezas quirúrgicas cubiertas totalmente por formol al $10 \%$, a fin de garantizar su adecuada conservación.

Criterios de exclusión:

- Piezas quirúrgicas desecadas por falta de formol o mal conservadas.

- Piezas quirúrgicas fragmentadas, desgarradas, incompletas o totalmente gangrenadas.

\section{Definiciones operacionales}

Colesterolosis: presencia, macroscópicamente, de puntos o estrías amarillentas en la superficie mucosa, que microscópicamente corresponden a presencia de macrófagos espumosos en la lámina propia de la mucosa de la vesícula biliar (figura 1).

Colecistolitiasis: Presencia de cálculos en el interior de la vesícula biliar.

Hipercolesterolemia: Colesterol total en sangre mayor de $200 \mathrm{mg} / \mathrm{dl}$.

\section{Análisis e interpretación de la información}

Se elaboraron tablas de 2x 2. Se utilizó Microsoft Excel 2010. Se utilizó la prueba de Chi cuadrado para determinar asociación de variables.

El estudio fue aprobado por el comité de ética del hospital. Es de notar que nuestro comité de ética 


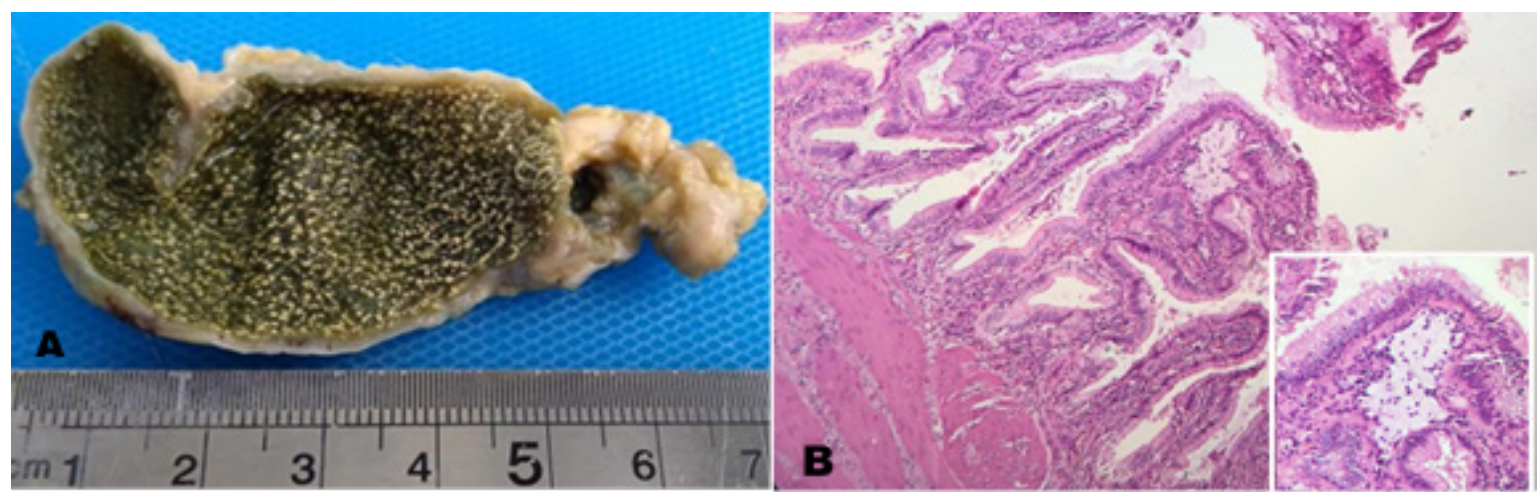

Figura 1. Colesterolosis. A: Vista macroscópica. La mucosa vesicular muestra innumerables gránulos amarillentos. B: Histológicamente corresponden a conglomerados de histiocitos en los pliegues mucosos (H/E, 100X). Recuadro: Magnificación del pliegue mucoso con conglomerado de histiocitos de citoplasma claro y núcleo pequeño cargados de colesterol en la lámina propia (H/E, 400X).

hospitalario aún no está acreditado por el Instituto Nacional de Salud.

\section{RESULTADOS}

Entre enero del 2014 y diciembre del 2015, en el Servicio de Patología Quirúrgica del Hospital Belén se registraron 1069 solicitudes de estudio anatomopatológicos de productos de colecistectomía. Las historias clínicas de estos pacientes fueron revisadas a fin de seleccionar aquellas que tuvieran perfil lipídico. De estas, sólo 143 casos (33 varones y 110 mujeres) formaron parte de este estudio debido a que contaban con perfil lipídico y reunían los criterios de inclusión. La edad de los pacientes osciló entre 17 y 95 años con una edad promedio de 50 años.

De los 143 casos, en $36(25,2 \%)$ se encontró colesterolosis, las restantes presentaron colecistitis.
La colesterolosis predominó en el sexo femenino (26 casos, 72,2\%), con una edad promedio de 44,5 años. La edad promedio de los 10 varones con colesterolosis fue 48,8 años. La relación mujer: varón fue 2,6:1. La colesterolosis fue más frecuente entre la 3ra y 5ta década de la vida. Con respecto al promedio de edad, no hubo diferencia estadísticamente significativa ( $p>$ 0,05 ) entre mujeres y varones con colesterolosis.

En 51/143 se encontró hipercolesterolemia (tabla 1). De los 119 pacientes que tuvieron litiasis, 29 $(24,4 \%)$ presentaron colesterolosis (tabla 2). De los 36 casos con colesterolosis, $29(80,5 \%)$ se asociaron a cálculos y siete no tuvieron cálculos. De los 24 casos que no tuvieron cálculos sólo 7 tuvieron colesterolosis.

Ciento siete casos tuvieron colecistitis sin colesterolosis, 90 tenían cálculos y 17 no. Dieciséis casos tenían hipercolesterolemia y colesterolosis,

Tabla 1. Características de 143 pacientes con perfil lipídico. (HBT 2014-2015).

\begin{tabular}{lcccccc}
\hline \multirow{2}{*}{ Hallazgo } & \multicolumn{2}{c}{ MASCULINO } & \multicolumn{2}{c}{ FEMENINO } & \multicolumn{2}{c}{ TOTAL } \\
\cline { 2 - 7 } & $\mathbf{n}$ & $\mathbf{\%}$ & $\mathbf{n}$ & $\mathbf{\%}$ & $\mathbf{n}$ & $\mathbf{\%}$ \\
\hline Colecistolitiasis & 26 & $78,8 \%$ & 93 & $84,5 \%$ & 119 & $83,2 \%$ \\
Alitiasis & 7 & $21,2 \%$ & 17 & $15,5 \%$ & 24 & $16,8 \%$ \\
Colesterolosis & & & & & & \\
$\quad$ - Calculosa & 5 & $50,0 \%$ & 24 & $92,3 \%$ & 29 & $80,6 \%$ \\
$\quad$ - Acalculosa & 5 & $50,0 \%$ & 2 & $7,7 \%$ & 7 & $19,4 \%$ \\
Colesterol sérico & & & & & & \\
$\quad$ - 2 200 mg/dl & 14 & $42,4 \%$ & 37 & $33,6 \%$ & 51 & $35,7 \%$ \\
$\quad$ - Normal & 19 & $57,6 \%$ & 73 & $66,4 \%$ & 92 & $64,3 \%$ \\
\hline
\end{tabular}


Tabla 2. Asociación entre hipercolesterolemia y colesterolosis o litiasis vesicular (HBT, 2014-2015).

\begin{tabular}{lcccccccc}
\hline Nivel de colesterol & \multicolumn{2}{c}{ Colesterolosis } & & \multicolumn{2}{c}{ Litiasis vesicular } & \multirow{2}{*}{ Total } \\
\cline { 2 - 3 } & Presente & Ausente & & & Presente & Ausente & & \\
\hline Hipercolesterolemia & 16 & 35 & 0,2844 & 39 & 12 & 0,1695 & 51 \\
Colesterol normal & 20 & 72 & & 80 & 12 & & 92 \\
Total & 36 & 107 & & 119 & 24 & & 143 \\
\hline
\end{tabular}

de ellos $12(75 \%)$ tenían $\mathrm{LDL} \geq 130 \mathrm{mg} / \mathrm{dl}$ y $50 \%$, hipertrigliceridemia $\geq 150 \mathrm{mg} / \mathrm{dl}$. Así mismo, de los 39 casos con hipercolesterolemia y colecistolitiasis, 35 $(89,7 \%)$ casos tuvieron $\mathrm{LDL} \geq 130 \mathrm{mg} / \mathrm{dl}, 28(71,8 \%)$ hipertrigliceridemia $\geq 150 \mathrm{mg} / \mathrm{dl}$ y $14(35,9 \%)$ casos $\geq 200 \mathrm{mg} / \mathrm{dl}$.

\section{DISCUSIÓN}

La colesterolosis en la mucosa de la vesícula biliar es el hallazgo más llamativo en los especímenes postcolecistectomía por litiasis sintomática. Esto nos motivó a investigar si la colesterolosis o la litiasis de la vesícula biliar, se asocian a hipercolesterolemia.

En el presente trabajo, la colesterolosis se observó en el $25,2 \%$ de todos los casos y asociada a colecistolitiasis en el 20,3\%. La colesterolosis, en casi todos los casos estuvo asociada a cambios mínimos de la mucosa vesicular y a escasa inflamación.

Con respecto a la edad, la mayor frecuencia estuvo entre los 30 y 59 años, muy similar a lo previamente reportado (16). Luego, disminuye hacia ambos extremos de la vida. Con respecto a su menor frecuencia antes de los 30 años, lo lógico es pensar que en las primeras décadas de la vida la colesterolosis y la colecistolitiasis todavía no se forman o recién se están formando. Lo difícil es encontrar una explicación para su menor frecuencia en las décadas después de los 60 años. A este respecto compartimos el punto de vista de Roa (17), quien plantea que para que se produzca la colesterolosis, el epitelio de la mucosa debe estar constituido por células normales que tengan "capacidad de concentrar e incorporar el colesterol presente en la bilis a través de un complejo sistema de receptores de membrana. La inflamación crónica y la litiasis vesicular producirían cambios regenerativos y adaptativos, los que conducen al reemplazo del epitelio propio por epitelio metaplásico", que ya no tendría capacidad de transportar colesterol. "A medida que progresa la intensidad de la metaplasia y la inflamación en la mucosa vesicular, se produciría la desaparición progresiva de la colesterolosis" (17).

Con respecto al sexo, al igual que en otros trabajos $(12,17,18)$, fue más frecuente en el sexo femenino. Con respecto a la colecistolitiasis, se considera factor predisponente el hecho de ser mujer. Esto debido a las hormonas estrogénicas, ya que estas aumentan las lipoproteínas de baja densidad (LDL, por sus siglas en inglés) al igual que las lipoproteínas de muy baja densidad (VLDL) y el colesterol en el jugo biliar y disminuyen dentro del mismo la concentración de ácidos y sales biliares lo que facilita la formación de litos dentro de la vesícula (13).

En nuestro estudio encontramos hipercolesterolemia en sólo el 44,4\% de los casos con colesterolosis. Un estudio hecho en Arabia Saudita (12), encontró hipercolesterolemia en el $85,1 \%$ de los casos con colesterolosis, concluyendo que existe una correlación positiva entre colesterolosis y altos niveles de colesterol en sangre. En el presente estudio, al aplicar las pruebas estadísticas, no encontramos una asociación estadísticamente significativa. Esta diferencia podría deberse a que la muestra de ellos fue el doble (74 casos) que la nuestra. Sin embargo, tomando en cuenta otros estudios $(7,15)$, consideramos que la colesterolosis es una manifestación poco frecuente de niveles elevados de colesterol y que podría haber otros factores aún no precisados que estarían condicionando esta variabilidad de resultados.

También, se encontró que el 80,5\% de los casos de colesterolosis presentaba concomitantemente litiasis y los restantes tenían colesterolosis acalculosa. Otros estudios $(12,19)$, también reportan estas diferencias de porcentaje. Esta disparidad en los hallazgos deja entrever que, aunque tanto la colescistolitiasis como la colesterolosis tienen mecanismos etiológicos similares, las dos lesiones pueden presentarse en forma independiente y no siempre coexisten en el mismo individuo. Tanto en la colecistolitiasis como en 
la colesterolosis se puede encontrar bilis sobresaturada de colesterol (16).

Con respecto al diagnóstico, Jacyna (19), sugiere que la colesterolosis debe ser considerada en aquellos pacientes con síntomas sugestivos de enfermedad biliar que no presentan anormalidades en la colecistografía ni en la ultrasonografía.

Con respecto a la colecistolitiasis, sólo en $32,8 \%$ hubo hipercolesterolemia. MéndezSánchez et al. (7), encontraron una asociación estadísticamente significativa entre colecistolitiasis e hipercolesterolemia. Sin embargo, en el presente trabajo, no se encontró asociación significativa. Aun cuando no hemos encontrado estudios que asocien la hipertrigliceridemia a la colesterolosis, nosotros encontramos hipertrigliceridemia mayor de $150 \mathrm{mg} /$ $\mathrm{dl}$, en el $71,8 \%$ de los casos con hipercolesterolemia.

La principal limitación que encontramos en el presente estudio fue el poco número de casos que tenían perfil lipídico. Consideramos que es necesario ampliar el periodo de estudio a fin de que este trabajo pueda tener conclusiones extrapolables. En conclusión, no se encontró asociación de hipercolesterolemia con colesterolosis ni con litiasis vesicular.

\section{Declaración de financiamiento y de conflictos de interés:}

Los autores declaran mediante juramento, que el estudio fue financiado por los autores, y declaran no tener conflictos de intereses con persona o institución alguna, ni de orden económico, ni institucional, ni laboral, ni personal.

\section{Contribución de autoría:}

CDVD: Diseño del estudio, estudio histológico, análisis e interpretación de los resultados, redacción, revisión crítica del artículo. GMAZ: Revisión de historias clínicas, control macroscópico, recolección de datos, documentación fotográfica y aprobación de la versión final a ser publicada. MJAV: Revisión de historias clínicas, control macroscópico, recolección de datos, referencias bibliográficas, aprobación de la versión final a ser publicada.

\section{Correspondencia:}

Carlos Vilela Desposorio

Baca Flor 346 Urb. Sto. Dominguito. Trujillo, Perú.

Correo electrónico: crlsvilela@yahoo.com

Teléfono: 5144474748 /51 948624929

\section{REFERENCIAS BIBLIOGRÁFICAS}

1. Weedon D. Cholesterolosis: Pathology of the gallbladder. New York: Masson; 1984. p. 161-165.

2. Zúñiga M, Barrantes M, Ugalde C. Pólipos de la vesícula biliar. Rev Med Costa Rica Centroamerica. 2013; 70(605):13-17.

3. Neiderhiser DH, Harmon CK, Roth HP. Absorption of cholesterol by the gallbladder. J Lipid Res. 1976; 17(2):117-24.

4. Jacyna MR, Ross PE, Bakar MA, Hopwood D, Bouchier IA. Characteristics of cholesterol absorption by human gallbladder: relevance to cholesterolosis. J Clin Pathol. 1987; 40(5):524-529.

5. Strömsten A, von Bahr S, Bringman S, et al. Studies on the mechanism of accumulation of cholesterol in the gallbladder mucosa. Evidence that sterol 27-hydroxylase is not a pathogenetic factor. J Hepatol. 2004; 40(1):8-13.

6. Sahlin S, Granström L, Gustafsson U, Ståhlberg D, Backman L, Einarsson K. Hepatic esterification rate of cholesterol and biliary lipids in human obesity. $\mathrm{J}$ Lipid Res. 1994; 35(3):484-490.

7. Méndez-Sánchez N, Tanimoto MA, Cobos E, RoldánValadez E, Uribe M. Cholesterolosis is not associated with high cholesterol levels in patients with and without gallstone disease. J Clin Gastroenterol. 1997; 25(3):518-521.

8. Liew PL, Wang W, Lee YC, Huang MT, Lin YC, Lee WJ. Gallbladder disease among obese patients in Taiwan. Obes Surg. 2007; 17(3):383-390.

9. Dittrick GW, Thompson JS, Campos D, Bremers D, Sudan D. Gallbladder pathology in morbid obesity. Obes Surg. 2005; 15(2):238-242.

10. Fobi M, Lee H, Igwe $\mathrm{D}$, et al. Prophylactic cholecystectomy with gastric bypass operation: incidence of gallbladder disease. Obes Surg. 2002; 12(3): 350-353.

11. Csendes A, Smok G, Burdiles P, Díaz JC, Maluenda F, Korn O. Histological findings of gallbladder mucosa in 95 control subjects and 80 patients with asymptomatic gallstones. Dig Dis Sci. 1998; 43(5):931-934.

12. Khairy GA, Guraya SY, Murshid KR. Cholesterolosis. Incidence, correlation with serum cholesterol level and the role of laparoscopic cholecystectomy. Saudi Med J. 2004; 25(9): 1226-1228.

13. Ivanchenkova RA, Sharashkina NV. Heterogeneidad de las lipoproteinas de baja densidad en la colesterolosis de la vesicular y colelitiasis. Klin Med. 
(Mosk) 2004; 82(10):46-49.

14. De la Cruz J. Indicaciones de la colecistectomía en la cirugía de la obesidad mórbida. Tesis doctoral. Barcelona: Universidad Autónoma de Barcelona; 2001, págs. 190.

15. Beltrán M, Barría C, Cruces K, Contreras M, Gonzáles F, Margulis D. Asociación de la colesterolosis vesicular con el índice de masa corporal y los niveles plasmáticos de colesterol. Gastroenterol Latinoam. 2010; 21 (4):432-436.

16. Jacyna MR, Bouchier IA. Cholesterolosis: a physical cause of "functional" disorder. Br Med J (Clin Res Ed). 1987; 295:619-620.

17. Roa E, De Aretxabala X, Ibacache G, Muñoz S. Colesterolosis y cáncer de la vesícula biliar Rev Med
Chile. 2010; 138(7):804-808.

18. Kmiot WA, Perry EP, Donovan IA, et al. Cholesterolosis in patients with chronic acalculous biliary pain. Br J Surg. 1994; 81(1):112- 115.

19. Nahum MS, Miguel AT, Cobos E, Ernesto RV, Uribe M. Cholesterolosis is not associated with high cholesterol levels in patients with and without gallstone disease. J Clin Gastroenterol. 1997; 25: 518-521.

Recibido: 01/11/2018

Aceptado: 20/12/2019 